\title{
Comment to Heng Luo et al.: Holmium laser division of the suture causing entrapment of a drainage tube after laparoscopic nephrectomy
}

\author{
Sudhir Khanna
}

Received: 6 April 2013/Accepted: 19 April 2013/Published online: 18 May 2013

(C) Springer Science+Business Media Dordrecht 2013

Editor,

I read with interest this article, which was a good application of laser.

I would have appreciated if the authors would have given a surface photograph of the patient's incision and the drain, to understand the problem better. It's not easy to understand how a suture for the main wound would go through the drain and that too $3 \mathrm{~cm}$ below the skin.

The authors have mentioned that they cut the thread with laser. They forgot to remove the cut end of the thread with the forceps. It would have been better to remove the cut ends of the thread with the help of forceps, why leave a non-absorbable thread. Removing the tube can inadvertently leave the thread.

This is an uncommon situation. How come they did not do any other investigation and jumped to this conclusion of suture and removed it straightaway?

Thanks

S. Khanna $(\bowtie)$

Urology, Sir Ganga Ram Hospital,

New Delhi, India

e-mail: sk1957@gmail.com 